UNITED STATES DEPARTMENT OF THE INTERIOR

GEOLOGICAL SURVEY

\title{
Analytical results and sample locality map \\ of winter moose pellet samples \\ from the Tanacross quadrangle, Alaska
}

By

G. W. Day, G. C. Curtin, R. B. Tripp, and J. S. Lewis

Open-File Report 85-268

This report is preliminary and has not been reviewed for conformity with U.S. Geological Survey editorial standards and stratigraphic nomenclature. Any use of trade names is for descriptive purposes only and does not imply endorsement by the USGS. 
$z z z z z z z z \quad z z z 0$

$z 22 z 2$

$z z z 2 z \quad z 2 z z=$

$2 z 22$

2222

$z \geq z \geq z$

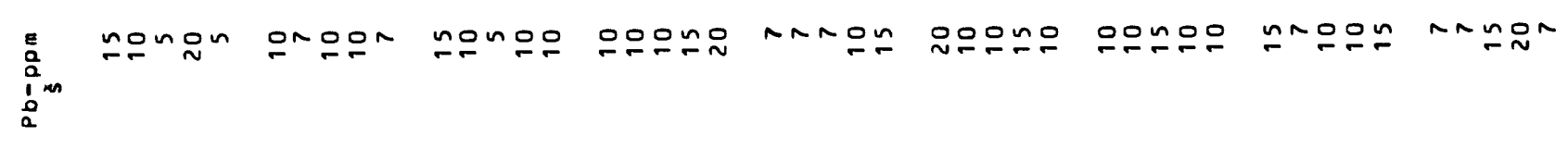

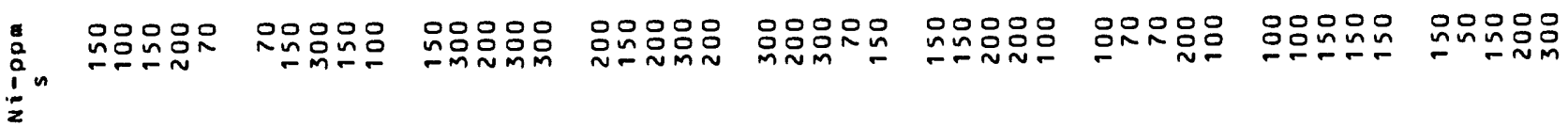

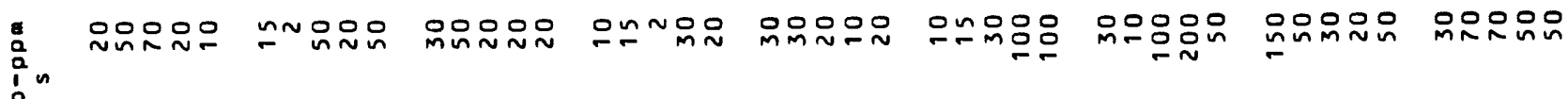
을

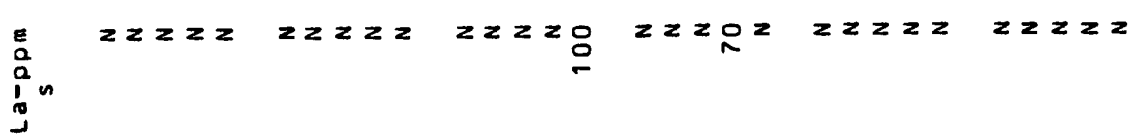

$z \geq z \geq 2$

$z \geq 2 z=$

$z \geq z z$

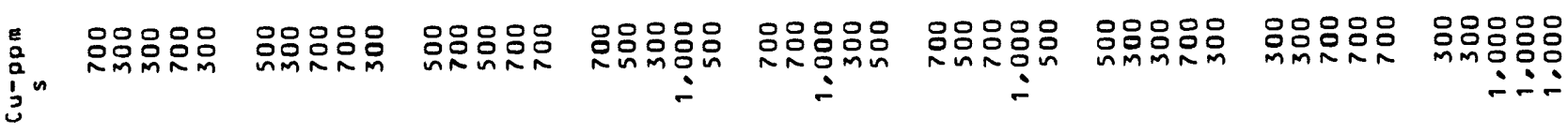

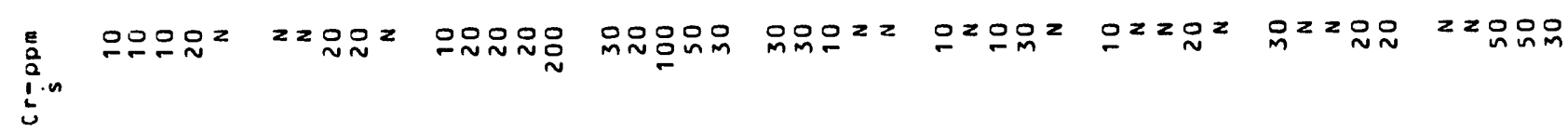

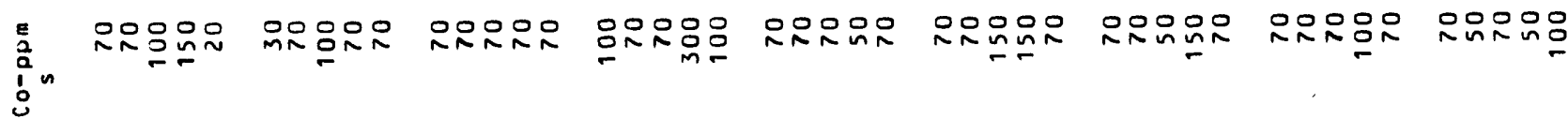

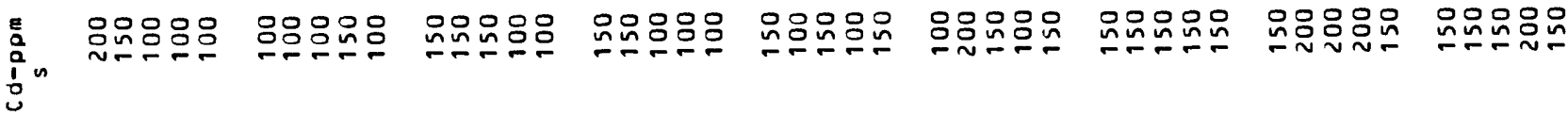




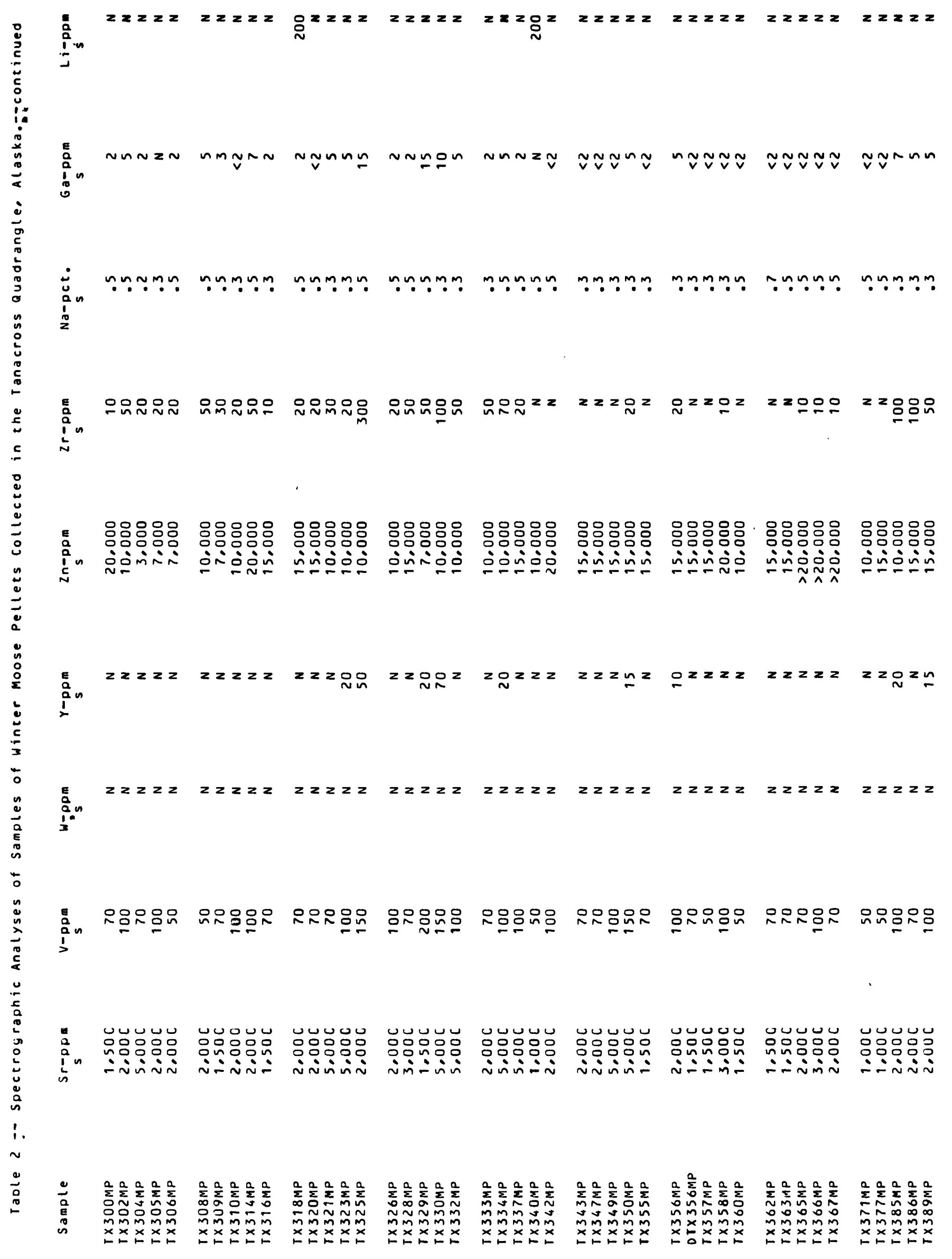




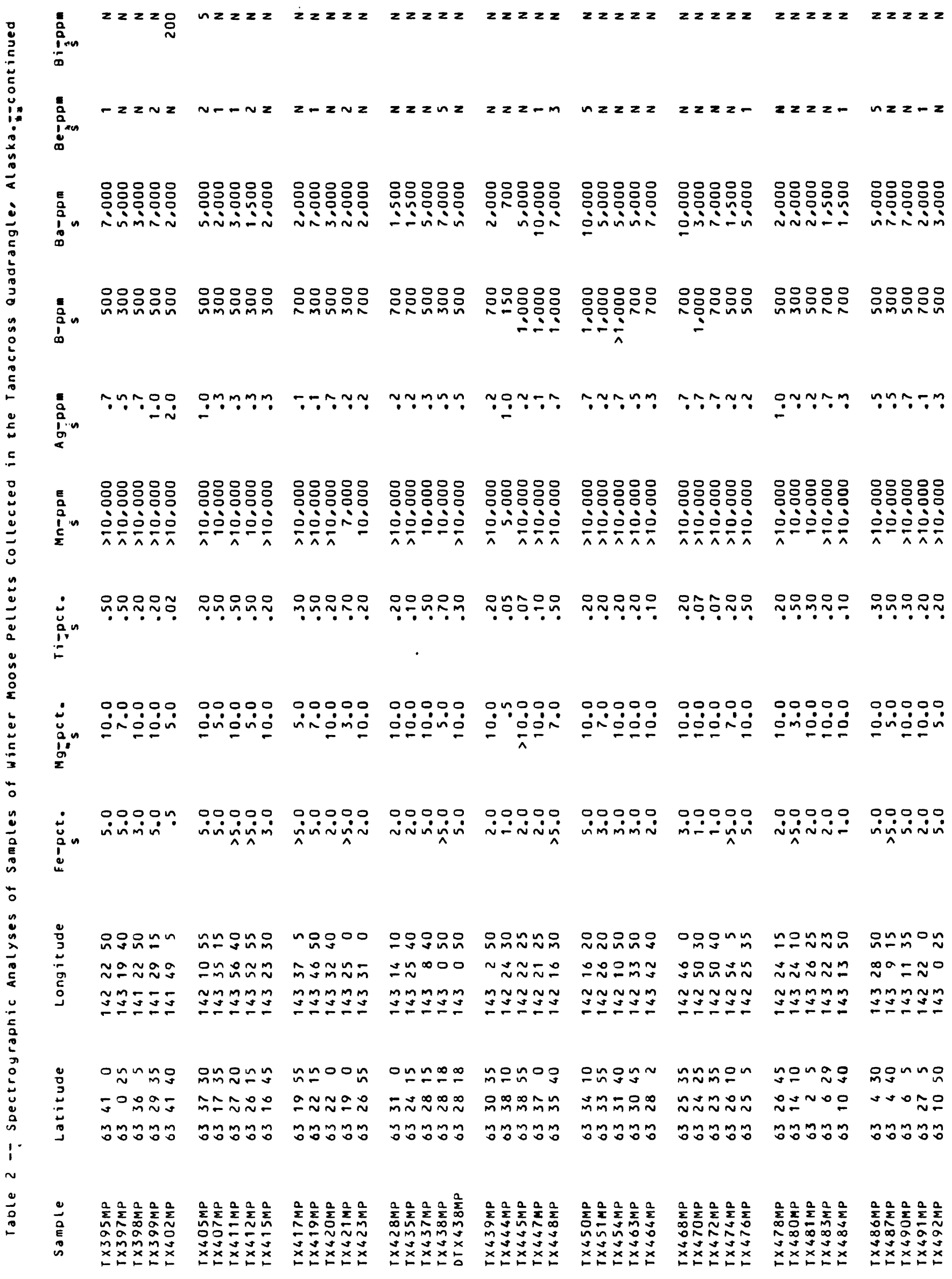




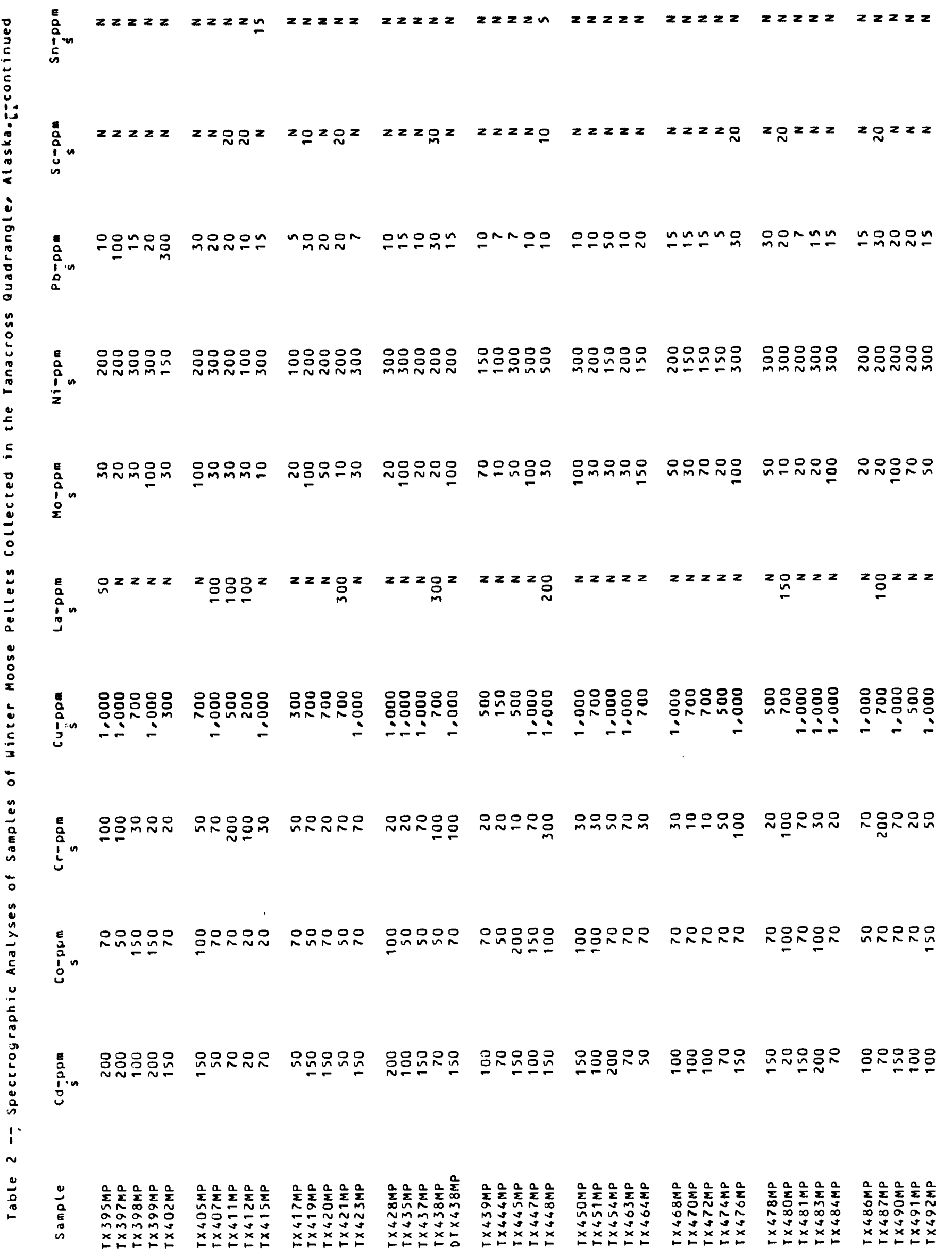




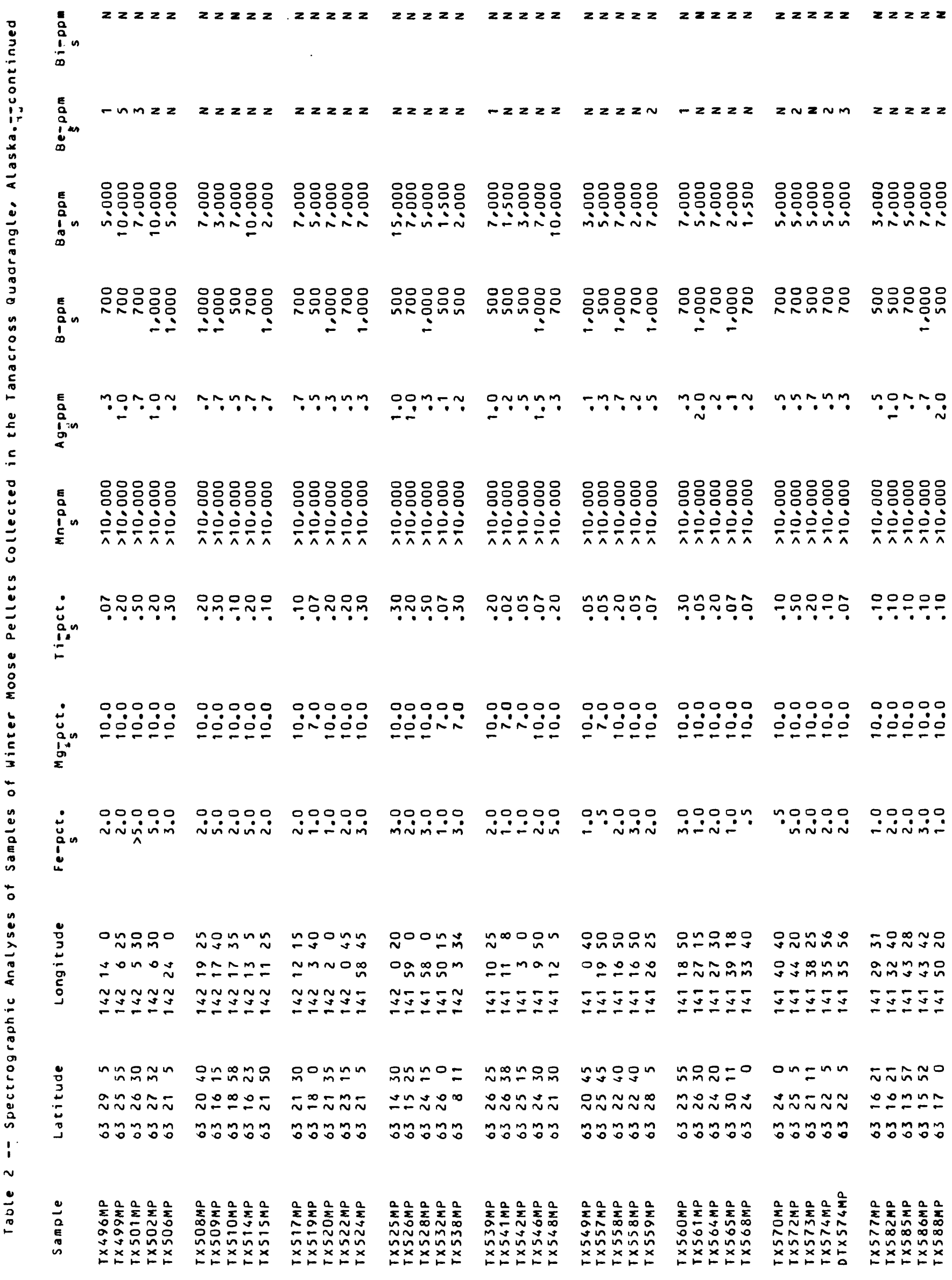




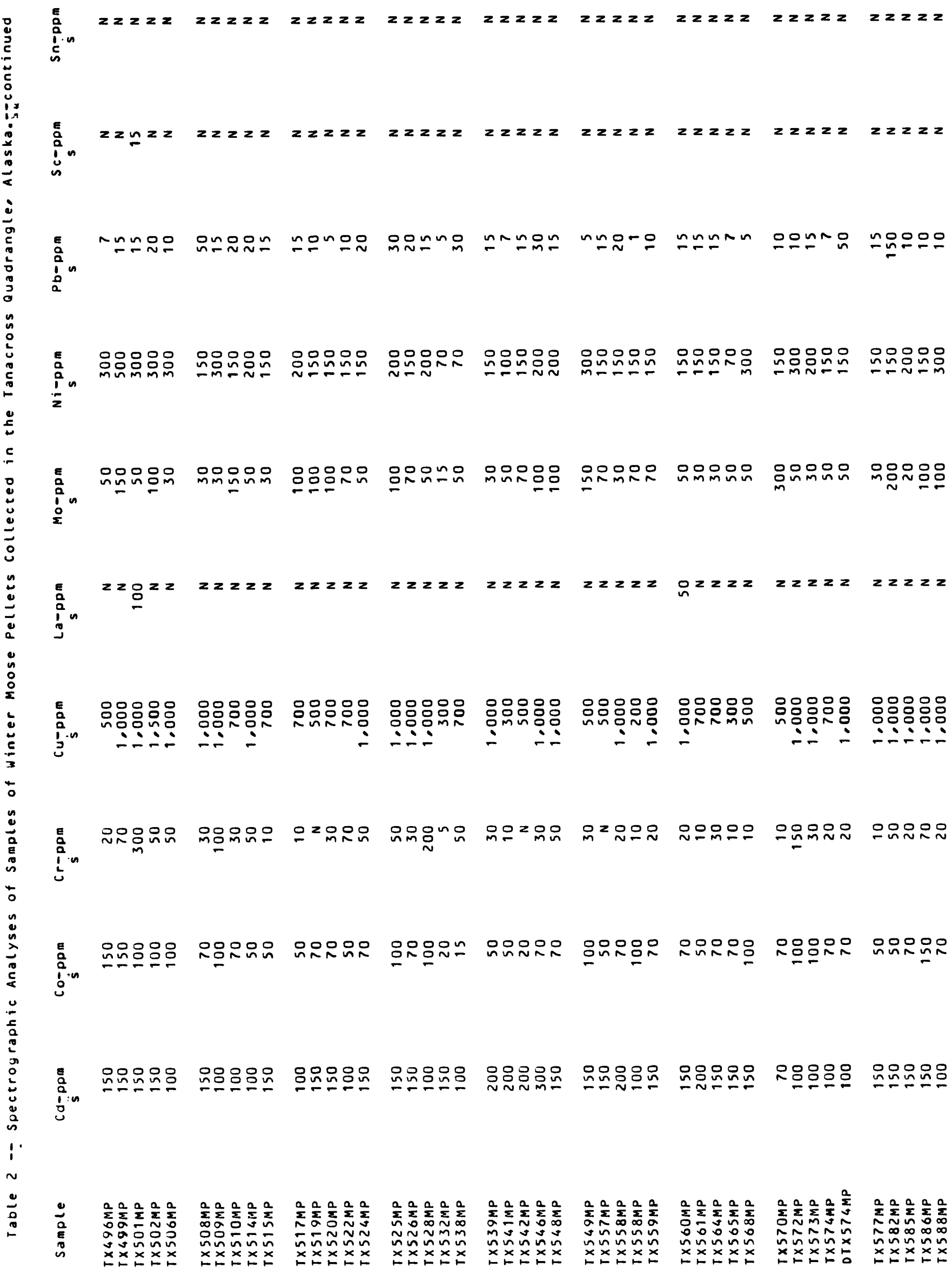




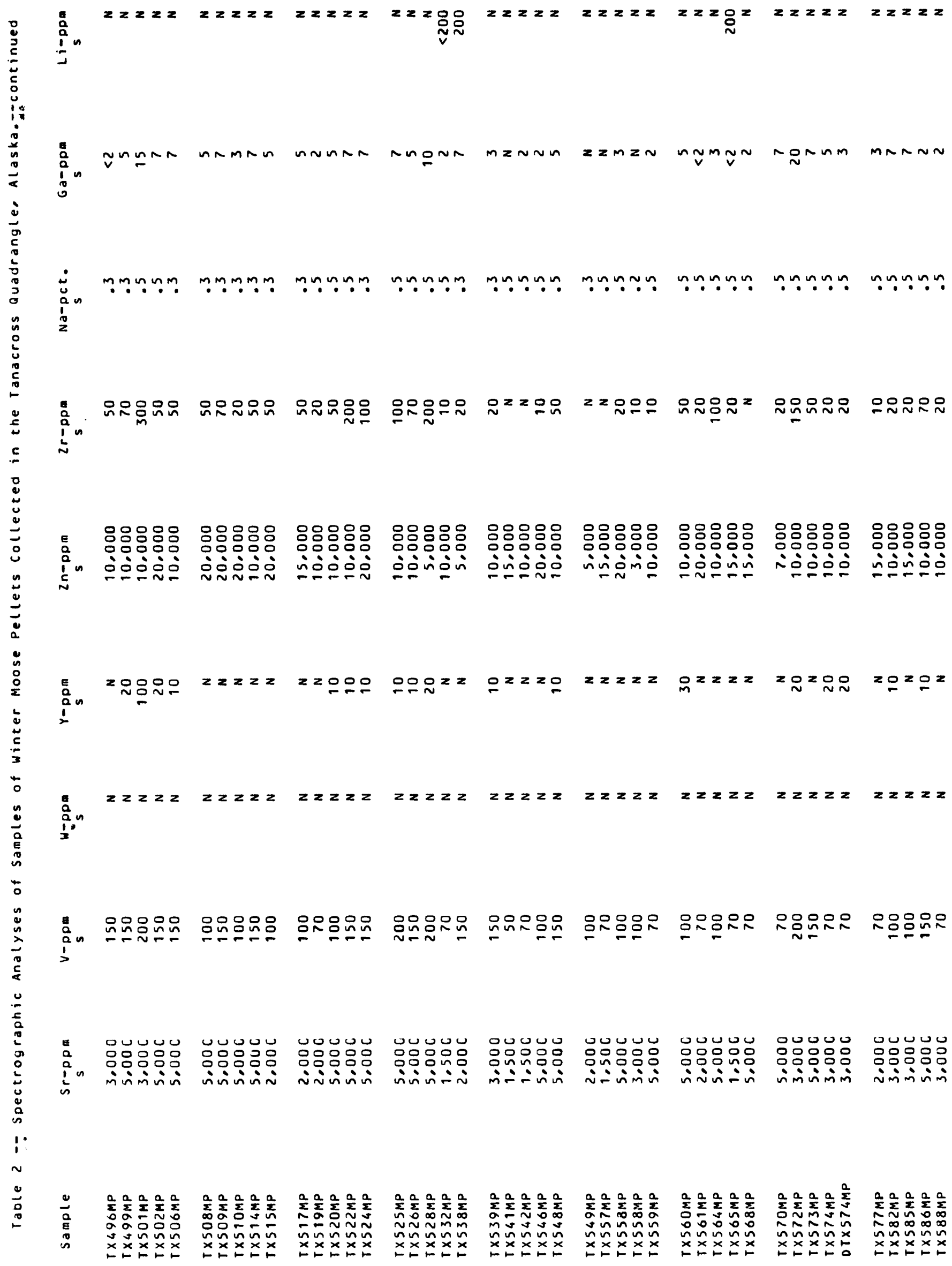




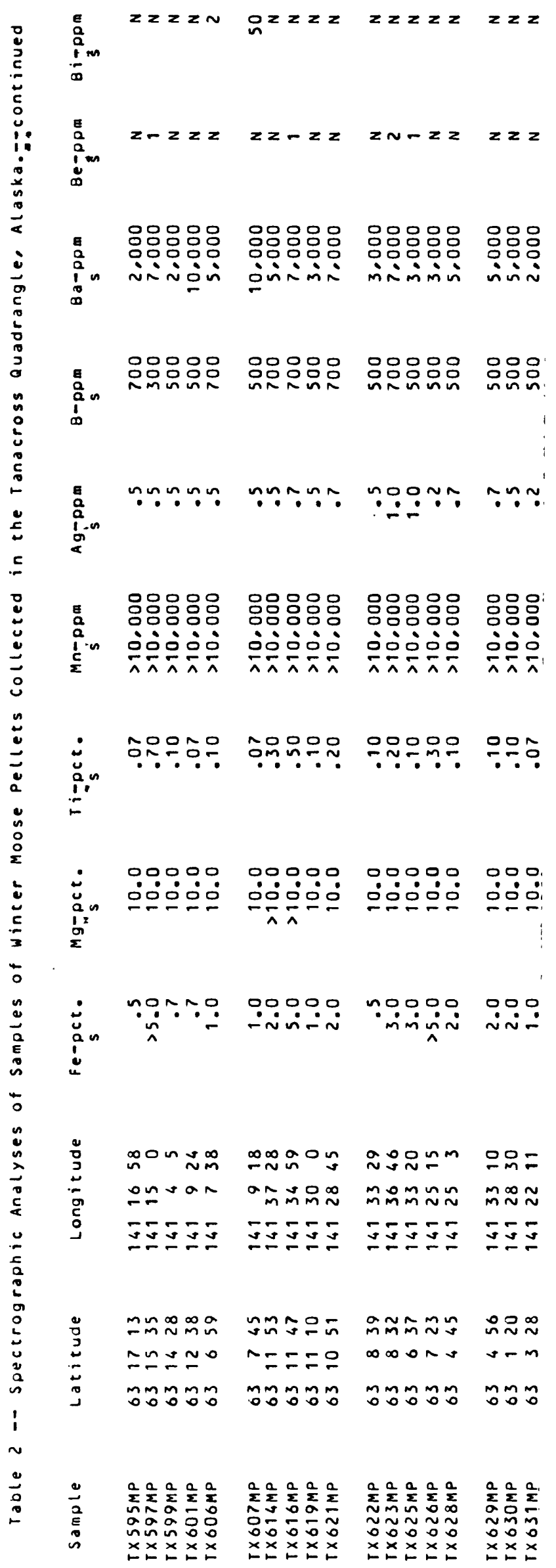




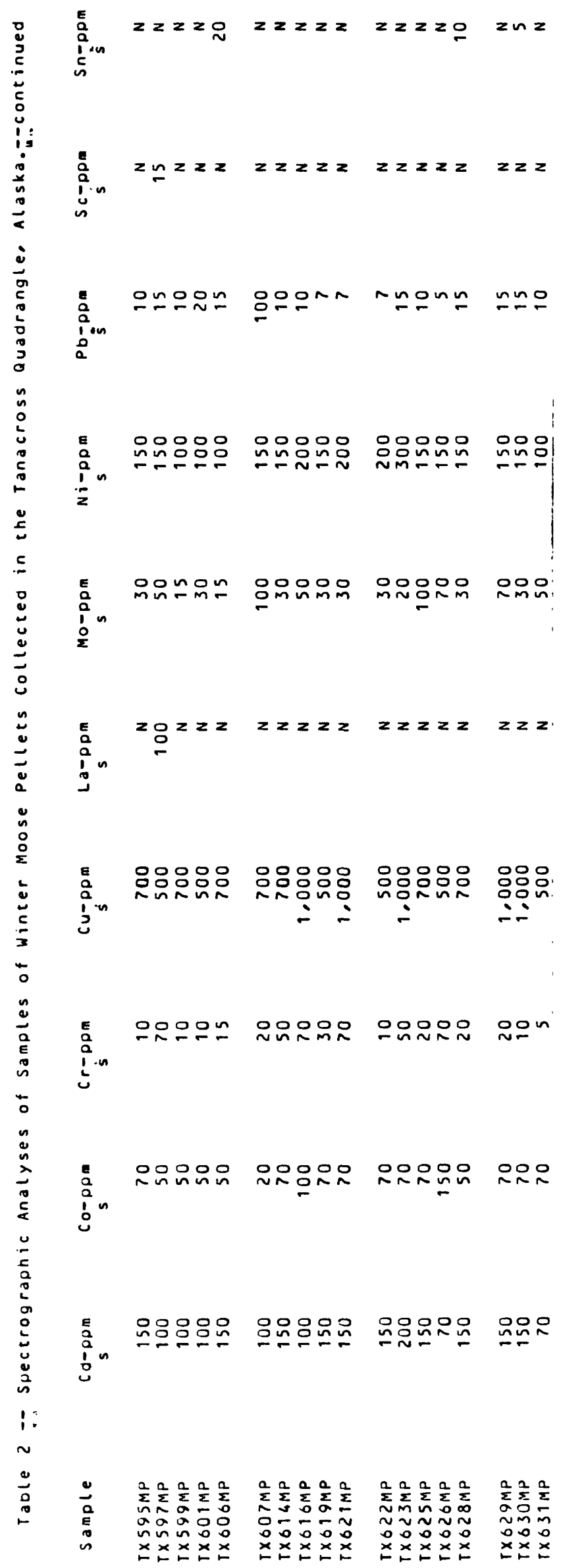




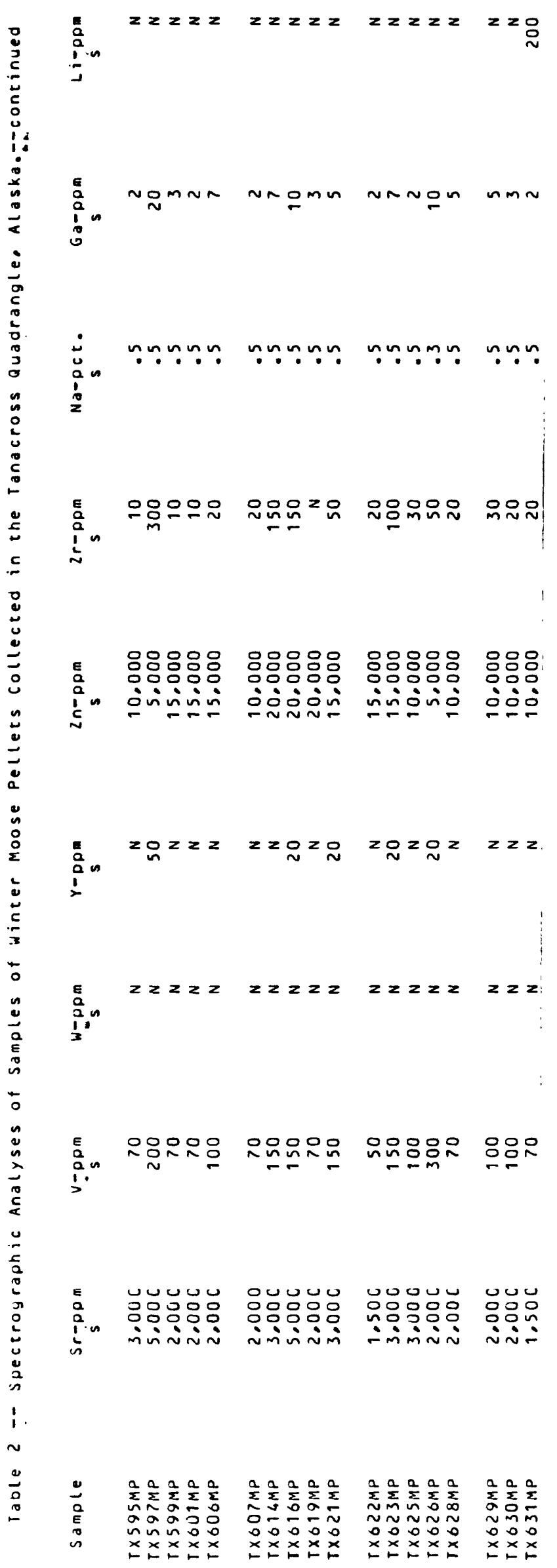


Table 3. Spectrographic analyses of elements found in three or less winter moose pellet samples

El ements not analyzed: Ca

Elements looked for but not found: Au, As, Ge, In, Tl

The following elements were not detected except in the samples listed:

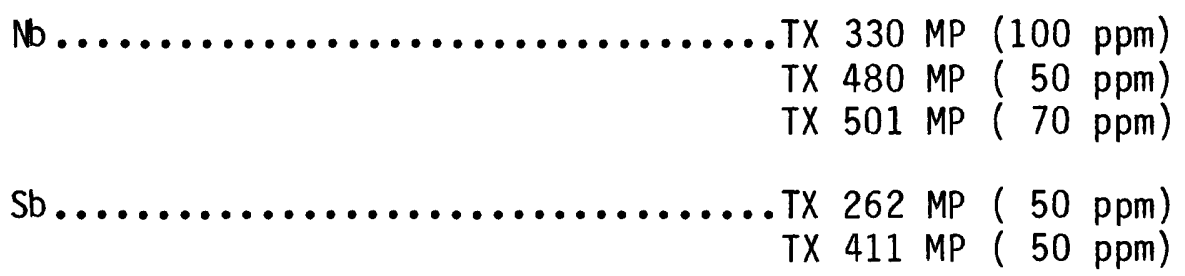

